Vassilios I. Vougioukas

Sven Gläsker

Ulrich Hubbe

Ansgar Berlis

Heymut Omran

Hartmut P. H. Neumann

Vera Van Velthoven

\title{
Surgical treatment of hemangioblastomas of the central nervous system in pediatric patients
}

\begin{abstract}
Objective: Hemangioblastomas are histologically benign lesions that occur sporadically or as a manifestation of von Hippel-Lindau disease (VHL). The treatment strategy of these neoplasms is complicated by their unpredictable growth patterns and the often irreversible neurological deficits they may cause. This study aims to outline the neurosurgical treatment options and to address the ongoing debate of surgical timing in pediatric patients with VHL. Patients and methods: Thirteen consecutive pediatric patients (mean age 15.1 years) who were surgically treated for intracranial $(n=8)$ or spinal hemangioblastomas $(n=5)$ were included in this study (range of clinical follow up 12-86 months). Ten patients were affected by von HippelLindau and three were with sporadic tumors. Serial examinations, preoperative MRI studies, and operative findings were reviewed. Results: Patients with cerebellar and intramedullary hemangioblastomas did not
\end{abstract}

develop additional neurological deficits postoperatively. Two patients with brainstem tumors exhibited transient hemiparesis and caudal nerve palsy, respectively. Both patients recovered completely from their postoperative deficits. Preoperatively symptomatic patients with spinal tumors did not deteriorate nor improve after surgery. During the observed follow-up periods, no tumor recurrences were observed. Conclusion: Central nervous system (CNS) hemangioblastomas in pediatric patients can be surgically treated with low morbidity. Based on our experience, we recommend considering also the surgical removal of asymptomatic hemangioblastomas with proven radiological progression to prevent the development of permanent neurological deficits. Molecular screening of every pediatric patient and family is mandatory to enable the detection of extraneurological tumors and the development of an efficient therapeutic strategy.

\section{Introduction}

Hemangioblastomas are highly vascular benign lesions that represent $3 \%$ of all central nervous system (CNS) tumors $[2,5,11]$. They occur as sporadic lesions or, in $50 \%$ of the cases, as component of von Hippel-Lindau disease (VHL), an autosomal dominant condition that results from germline mutations on chromosome 3 and predisposes individ- uals to the development of a variety of malignant and benign neoplasms [7]. Other disease manifestations include renal cysts and renal carcinoma, pancreatic cysts, neuroendocrine tumors, cystadenomas of the reproductive adnexal organs as well as hemangioblastomas of the cerebellum, spinal cord, brain stem, peripheral nerves and retina [8]. Despite the benign nature of CNS hemangioblastomas, it has been well documented that these tumors 
may cause significant irreversible neurological damage [9, $12,15]$. Recent advances in imaging and microsurgical techniques have clearly improved the overall hemangioblastoma-associated morbidity $[13,15,16]$. Several studies in the current literature outline treatment options in adult patients with CNS hemangioblastomas $[8,9,13,15,16]$. Nevertheless, there is still an ongoing debate concerning the treatment modality and timing of surgical intervention in such lesions $[1,3,6]$.

Overall, sporadic hemangioblastomas as well as VHL disease among patients under the age of 18 are extremely rare with an incidence of less than 1 per 1,000,000 [4, 14]. The average age at presentation is about 33 years in the VHL-related and 43 years in the sporadic cases [10]. Therefore, the occurrence of multiple hemangioblastomas at young age is highly suggestive of VHL.

There has been no comprehensive review of the surgical management of CNS hemangioblastomas in children and adolescents. To investigate the surgical outcome and to define surgical indications, we reviewed a series of 13 adolescent patients who underwent surgical resection of CNS hemangioblastomas and outline our treatment strategy in such complicated cases.

\section{Patients and methods}

Patient population

Between January 1988 and January 2003, a total of 141 patients with von Hippel-Lindau disease underwent microsurgical removal of CNS hemangioblastomas at the Neurosurgical Department of the University of Freiburg. We retrospectively analyzed clinical and radiological data for all patients under the age of 16 years. Table 1 summarizes the clinical data.

Neurological evaluation and surgical technique

Preoperative neurological status and postoperative outcome were assessed according to data obtained from medical charts and telephone interviews with the patients.

For cerebellar tumors and lesions of the craniocervical junction, a suboccipital osteoplastic craniotomy was performed. Intramedullary hemangioblastomas were approached via laminotomy or hemilaminectomy. The overlying arachnoidea was removed and the tumors were dissected circumferentially from the nervous tissue. Meticulous coagulation at low power and cutting of feeding vessels was essential for the bloodless en bloc removal of the tumor. The associated pseudocysts were automatically opened during tumor removal. Perioperative monitoring including somatosensory-evoked potentials, motor-evoked potentials, and cranial nerve monitoring was performed when necessary.

\section{Results}

Thirteen patients (seven male and six female, age range 916, mean age 15.1 years) were operated on CNS

Table 1 Clinical data of patients

\begin{tabular}{|c|c|c|c|c|c|}
\hline Patients & Location & $\begin{array}{l}\text { Age at } \\
\text { operation }\end{array}$ & VHL disease & Preoperative symptoms & Outcome \\
\hline G.A. & Cerebellum & 9 & DNA not tested & Ataxia & No deficit \\
\hline I.D. & TH 3 & 11 & Positive (DNA: insertion) & Paraparesis & $\begin{array}{l}\text { No additional } \\
\text { deficit }\end{array}$ \\
\hline R.K & Brainstem & 13 & DNA not tested & Headache, vomiting & $\begin{array}{l}\text { Temporary } \\
\text { hemiparesis }\end{array}$ \\
\hline N.S. & TH 7-9 & 14 & $\begin{array}{l}\text { Positive }(676+2 \text {, splice } \\
\text { mutation })\end{array}$ & Paraparesis & $\begin{array}{l}\text { No additional } \\
\text { deficit }\end{array}$ \\
\hline P.S. & C5 & 14 & Positive (10-kb deletion) & Radicular pain & No deficit \\
\hline R.S & $\mathrm{C} 3-5$ & 14 & Positive (437-439 deletion) & Radicular pain & $\begin{array}{l}\text { No additional } \\
\text { deficit }\end{array}$ \\
\hline G.B. & TH12 & 15 & Positive $(703 \mathrm{C} / \mathrm{T})$ & Asymptomatic & No deficit \\
\hline M.K. & Cerebellum & 15 & Negative (DNA: no mutation) & Ataxia & No deficit \\
\hline P. G. & Cerebellum & 15 & Positive $(694 \mathrm{C} / \mathrm{G})$ & Ataxia, GD & No deficit \\
\hline R.D & Cerebellum & 15 & Positive (437 in A) & Ataxia, GD & No deficit \\
\hline K.W & Brainstem & 16 & Negative (DNA: no mutation) & Asymptomatic & $\begin{array}{l}\text { Temporary IX, X } \\
\text { paresis and } \\
\text { tracheostomy }\end{array}$ \\
\hline M.R & Cerebellum & 16 & Positive (443+3 insertion) & Ataxia, hydrocephalus & No deficit \\
\hline R.A. & Cerebellum & 16 & Negative (DNA: no mutation) & Ataxia, hydrocephalus & No deficit \\
\hline
\end{tabular}



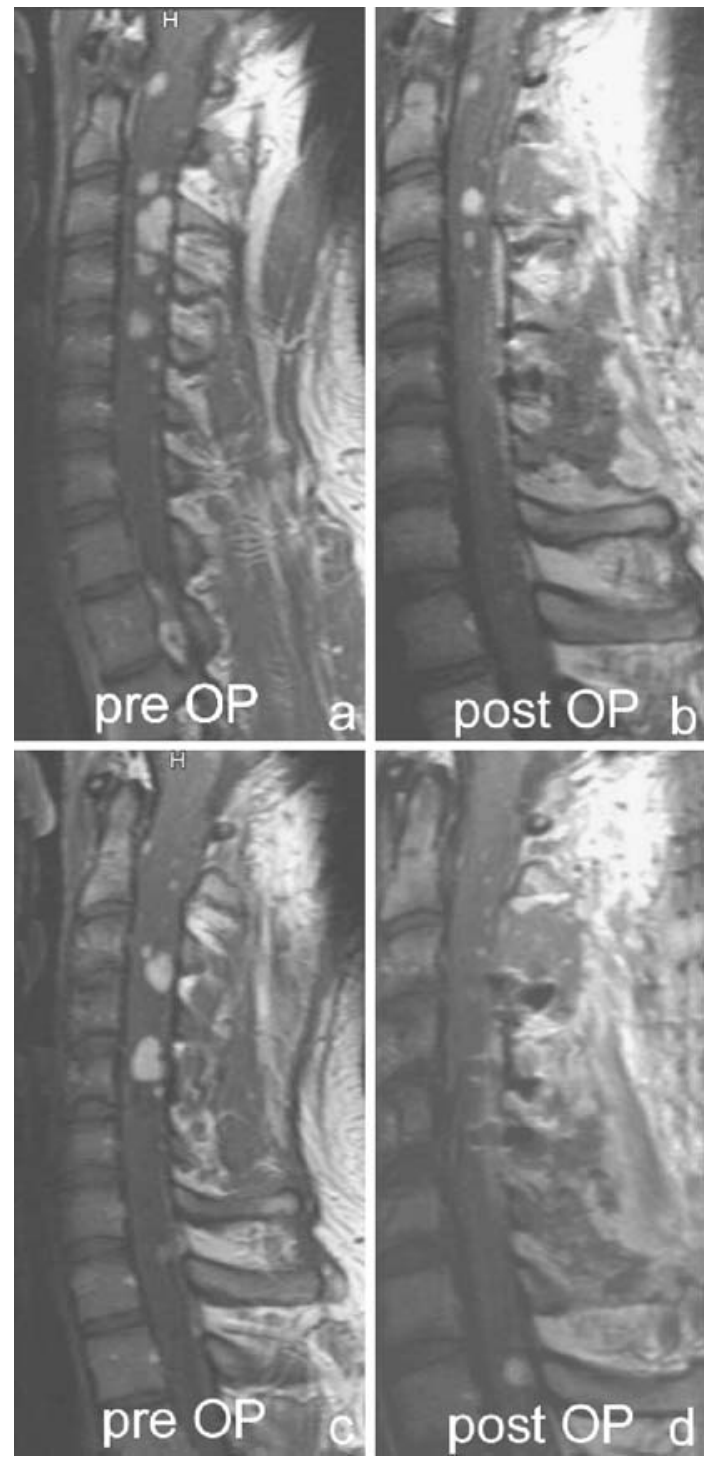

Fig. 1 a, c Pre-operative sagittal MRI contrast-enhanced images demonstrating multiple cervical hemangioblastomas. b, d Corresponding postoperative images after laminotomy C3-C5. 70×143 mm $(200 \times 200$ DPI $)$

Fig. 2 a Preoperative axial MRI image of the hemangioblastoma on the $\mathrm{C} 3 / 4$ level. b Corresponding postoperative image. $135 \times 59 \mathrm{~mm}$ $(200 \times 200 \mathrm{DPI})$ hemangioblastomas. Follow-up periods ranged from 12 to 86 months (mean 24.6 months).

Three patients had sporadic tumors; the other ten were affected with VHL. Genetic screening for mutations or deletions of the VHL tumor suppressor gene was performed on eight of the ten VHL patients and all three sporadic patients. The eight VHL patients revealed different mutations and deletions, whereas no mutations were identified in the patients with sporadic tumors (Table 1).

Six patients were treated for cerebellar lesions, five patients were operated on intramedullary tumors, and, finally, in two cases brainstem hemangioblastomas were microsurgically resected. In 11 cases, patients presented with neurological symptoms that were attributed to tumor localization and extent. However, one patient with an intramedullary lesion and one child with a brainstem tumor were asymptomatic and were operated on due to radiologically validated tumor size progression.

Initial symptoms depending on tumor localization included pain $(n=2)$, cerebellar deficits $(n=7)$, motor disturbances $(n=3)$, and hydrocephalus $(n=2)$. All patients with cerebellar and intramedullary tumors did not exhibit any additional neurological symptoms after surgery. The two patients with brainstem hemangioblastomas in the craniocervical junction developed temporary hemiparesis and caudal cranial nerve palsy, respectively. Both patients recovered completely from their postoperative deficits. During the reported follow-up periods, there was no tumor recurrence in loco in any of the patients.

\section{Case illustration}

A 14-year-old male patient with genetically validated VHL disease presented to our clinic with severe myeloradiculopathy corresponding to the $\mathrm{C} 4-\mathrm{C} 5$ and $\mathrm{C} 6$ roots on the right side. Clinical examination revealed motor deficits of
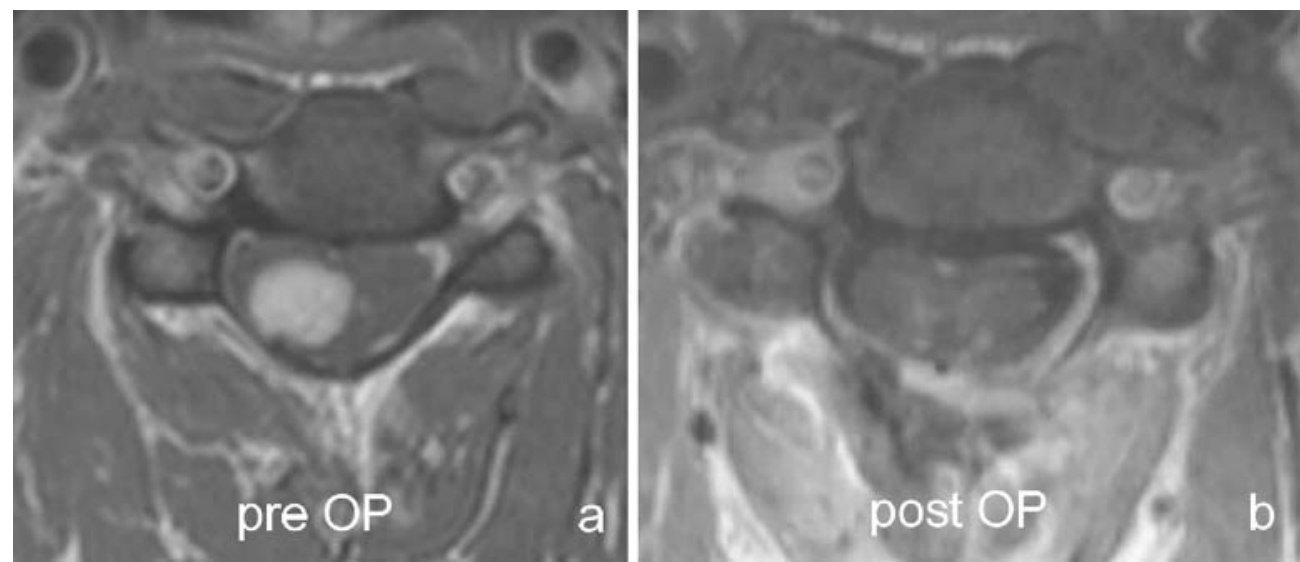
all muscle groups of the right arm (four out of five). Deep tendon reflexes were symmetrically increased and the gait was slightly ataxic. MRI images of the spine showed multiple hemangioblastomas throughout the cervical spinal cord (Figs. 1 and 2). The patient underwent laminotomy C3-C5 and removal of the clinically relevant tumors. Postoperative course was uneventful. Radicular pain subsided and the gait disturbance was unchanged. During the following 12 months, there was no tumor recurrence. The size of the untreated hemangioblastomas remains stable.

\section{Discussion}

The therapy for hemangioblastomas in adult patients is standardized and poses usually no major difficulties with the use of modern microneurosurgery $[8,15]$. However, in the context of VHL disease, even children and adolescents can develop these tumors. There is so far no knowledge about the adequate management of these patients. This uncertainty results from the lack of studies clarifying the surgical indications and outcome of this subset of patients. Furthermore, due to the unpredictable growth pattern of hemangioblastomas, the development of an efficient therapeutic strategy is absolutely essential to avoid the inherent neurological deficits that they may cause. Currently no definite guidelines concerning the surgical treatment of CNS hemangioblastomas in the pediatric and adolescent age group exist. The percentage of VHL patients with hemangioblastoma-associated morbidity decreases after the fourth decade of life. In addition, the presence of one or more hemangioblastomas in the pediatric age group is highly suggestive for VHL disease. In our series, 10 out of 13 patients had a germline VHL mutation. Positive genetic testing has significant consequences for further treatment planning. We recommend that every patient, especially in the pediatric age, with CNS hemangioblastoma should be screened for germline mutations of the VHL gene. Patients with positive genetic findings should undergo a general screening program, including contrast-enhanced MRI studies of the cranium and spine, CT or MRI evaluations of the abdomen, ophthalmological examination, urine analyses for determination of catecholamine excretion, and urological examinations. These examination series should be repeated on an annual basis to detect tumor progression.

In general, symptom production in hemangioblastomas is a product of lesion size and location [17]. In most of the cases, symptoms are related to pseudocysts associated with the tumor. The exact pathogenesis of the tumor-associated pseudocysts is not known. It has been hypothesized that transudation of fluid from the tumor capillaries and tubular dissection along the gray matter near the central canal are the main pathophysiological mechanisms [15]. It should be noted that the pseudocyst may be entered during microsurgical tumor resection, but additional removal of the cyst wall is not necessary. Nevertheless, complete removal of the solid tumor parts is mandatory to avoid tumor recurrence.

Children and adolescents with VHL syndrome may present with multiple tumors affecting all parts of the CNS, but mainly cerebellum, brainstem and spinal cord. It is often impossible to cure these patients from all their lesions. Nevertheless, the development of a treatment strategy is essential to minimize neurological damage. Staged procedures are quite common for patients with multiple tumors. Furthermore, a considerable number of tumors remain stable for several years or may even never show signs of progression. Thus, we recommend surgery only in cases with symptomatic lesions or tumors with validated progression. Care should be taken in patients with multiple laminotomies due to the development of spinal instability or cord tethering.

It is generally accepted that postoperative outcomes especially for intramedullary and brain stem tumors highly depend on the preoperative neurological status $[9,15,16]$. Thus, from our point of view, validated radiological tumor growth even in patients without complaints justifies neurosurgical intervention. Despite the extremely low morbidity, as demonstrated in the present study, it is mandatory to obtain a detailed informed consent especially in asymptomatic cases. Patients should understand that a conservative treatment strategy especially in the case of tumors with validated radiological growth may lead to irreversible deficits and that surgical treatment represents an effective and safe treatment when performed by an experienced surgeon.

\section{Conclusion}

Our results demonstrate that CNS hemangioblastomas can be removed with very low surgical morbidity, depending on tumor location, when surgery is performed before significant disability occurs. Surgical timing especially in VHL patients with multiple tumors remains a matter of debate. We recommend surgical removal of symptomatic and asymptomatic tumors with validated radiological size progression. Children with both positive or negative family history and positive genetic testing for a VHL germline mutation should undergo an extensive medical, radiological, ophthalmological, neurological and urological screening. 


\section{References}

1. Chang SD, Meisel JA, Hancock SL et al (1998) Treatment of hemangioblastomas in von Hippel Lindau disease with linear accelerator based radiosurgery. Neurosurgery 43:28-35

2. de la Monte SA, Horrowitz SA (1989) Hemangioblastomas: clinical and histopathological factors correlated with recurrence. Neurosurgery 25:695-698

3. Eskridge JM, McAuliffe W, Harris B (1996) Preoperative endovascular embolisation of craniospinal hemangioblastomas. AJNR Am J Neuroradiol 17:525-531

4. Fisher PG, Tontiplaphol A, Pearlman EM et al (2002) Childhood cerebellar hemangioblastoma does not predict germline or somatic mutations in the von Hippel-Lindau disease. Ann Neurol 51:257-260

5. Glasker S, Bender BU, Apel TW et al (1999) The impact of genetic molecular analysis of the VHL gene in patients with hemangioblastomas of the central nervous system. J Neurol Neurosurg Psychiatry 67:758-762
6. Hermann HD, Neuss M, Winkler D (1988) Intramedullary spinal cord tumors resected with $\mathrm{CO}_{2}$ laser microsurgical technique: recent experience in fifteen patients. Neurosurgery 22:518522

7. Latif F, Tory K, Gnarra J et al (1993) Identification of the von Hippel-Lindau disease tumor suppressor gene. Science 260:1317-1320

8. Lonser RR, Glenn GM, Walther $\mathrm{M}$ et al (2003) von Hippel-Lindau disease. Lancet 361:2059-2067

9. Lonser RR, Weil RJ, Wanebo JE (2003) Surgical management of spinal cord hemangioblastomas in patients with von Hippel-Lindau disease. J Neurosurg 98:106-116

10. Maher ER, Yates JRW, FergusonSmith MA (1990) Statistical analysis of the two stage mutation model in von Hippel-Lindau disease, and in sporadic cerebellar hemangioblastoma and renal cell carcinoma. J Med Genet 27:311-314

11. Neumann HP, Eggert HR, Scheremet R et al (1992) Central nervous system lesions in von Hippel-Lindau syndrome. J Neurol Neurosurg Psychiatry 55:898-901

12. Neumann HP, Eggert HR, Weigel $K$ et al (1989) Hemangioblastomas of the central nervous system: a 10-year study with special reference to the von Hippel-Lindau syndrome. J Neurosurg 70:24-30
13. Roonprapunt C, Silvera VM, Setton A et al (2001) Surgical management of isolated hemangioblastomas of the spinal cord. Neurosurgery 49:321-328

14. Ries LAG, Eisner MP, Kosary CL et al (2001) SEER cancer statistics review, 1973-1998. National Cancer Institute, Bethesda, MD

15. Van Velthoven V, Reinacher PC, Klisch $\mathrm{J}$ et al (2003) Treatment of intramedullary hemangioblastomas, with special attention to von Hippel-Lindau disease. Neurosurgery 53:1306-1314

16. Weil RJ, Lonser RR, DeVroom HL et al (2003) Surgical management of brainstem hemangioblastomas in patients with von Hippel-Lindau disease. J Neurosurg 98:95-105

17. Wanebo JE, Lonser RR, Glenn GM et al (2003) The natural history of hemangioblastomas of the central nervous system in patients with von Hippel-Lindau disease. J Neurosurg 98:82-94 